Supporting Information for

\title{
Hydantoin and Its Derivatives Reduce the Viscosity of Concentrated Antibody Formulations by Inhibiting Associations via Hydrophobic Amino Acid Residues
}

Suguru Nishinami ${ }^{1}$, Tomoshi Kameda ${ }^{2}$, Tsutomu Arakawa $^{3}$, Kentaro Shiraki $^{1 *}$

${ }^{1}$ Faculty of Pure and Applied Sciences, University of Tsukuba, 1-1-1 Tennodai, Tsukuba, Ibaraki 305-8573, Japan.

${ }^{2}$ Artificial Intelligence Research Center, Advanced Industrial Science and Technology (AIST), 2-4-7 Aomi, Koto, Tokyo 135-0064, Japan.

${ }^{3}$ a Division of KBI Biopharma, Alliance Protein Laboratories, San Diego, California 92121, United States.

* To whom correspondence should be addressed. Telephone: +81-29-8535306; Fax: +81-29-8535215; E-mail: shiraki@ bk.tsukuba.ac.jp

Figures
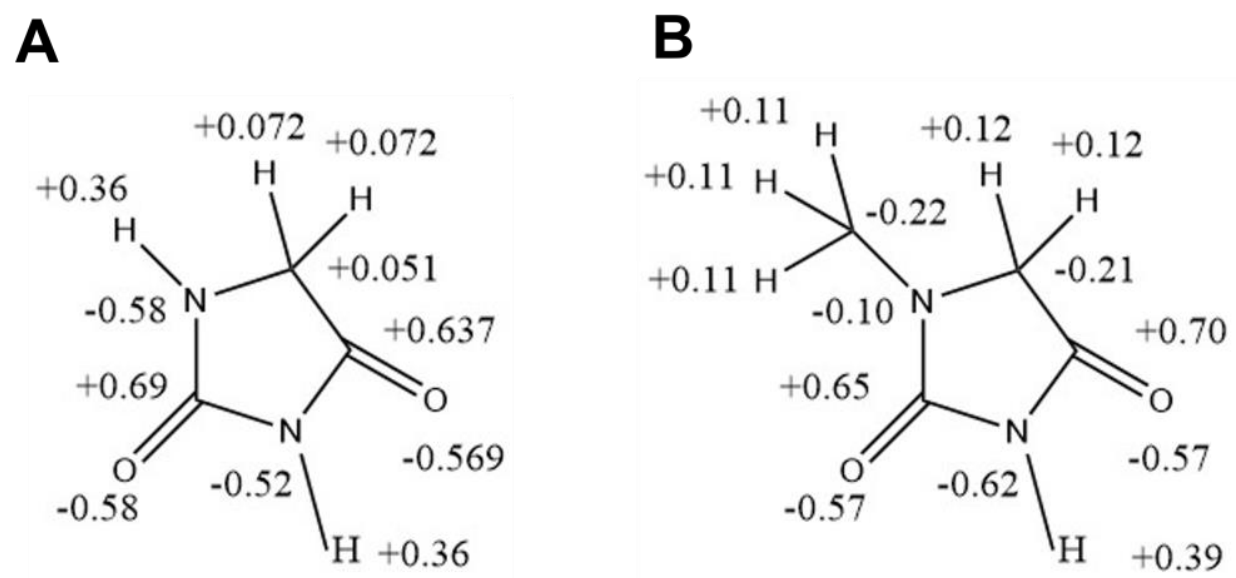

Figure S1. The partial charges of (A) hydantoin and (B) 1-methylhydantoin molecule. 